\title{
Underdetermined BSS of MISO OSTBC signals
}

\author{
A. Mansour ${ }^{1}$, J. Youssef ${ }^{2}$, and K.-C. Yao ${ }^{2}$ \\ 1 Depart. of ECE, Curtin University, Perth, WA 6152, Australia. \\ ${ }^{2}$ LEST UMR 6165, UBO, Brest, France \\ mansour@ieee.org; koffi-clement.yao@univ-brest.fr
}

\begin{abstract}
To improve the bit rate, the effectiveness of wireless transmission systems and limit the effects of fading transmission channel, an increased attention have recently been paid to MIMO systems. In fact, Alamouti's space-time block code is introduced in various wireless standards and systems. This manuscript deals with the problem of presence identification as well as blind separation of Orthogonal Space-Time Block Code (OSTBC) in the context of non data aided.

KEY WORD: MIMO channel, OSTBC, ICA, Alamouti's code, BSS of underdetermined mixture.
\end{abstract}

\section{Introduction}

In the last two decades, wireless communication systems become one of few attractive economical sectors and the most challenging technology issues facing worldwide engineering institutions. In modern society, wireless communication systems and gadgets are an important part of our every day life. Wireless systems are the most up-to-date electronic technology and they can be found in various applications such as: Remote control toys, Global Positioning System (GPS), Mobil phone, radio, TV, satellite, robotics, etc.

The actual mobil phone standards such as, GSM(Global System for Mobile communications), GPRS(General Packet Radio Service) and UMTS(Universal Mobile Telecommunications System), can't support a very high data rate. In addition, the transmission system reliability and the transmission quality depend much on channel conditions. To improve the overall transmission performance using the diversity of transmission channel, Multiple-Input Multiple Output (MIMO) transmission systems have recently been introduced by many researchers and engineers. In fact, these systems have shown their abilities to tackle fading effects in multipath channels $[15,4,9]$. MIMO systems use SpaceTime Block Coding (STBC) designs. According to that property, different STBC systems can be designed. In the literature, STBC which have an orthogonal coding matrix are the most used ones and they are called the Orthogonal STBC (OSTBC). Recently, OSTBC have been introduced in recent wireless communication technology such as Worldwide Interoperability for Microwave Access (WiMax), and IEEE Standard 802.16e for 2.5GHZ bands.

One of the possible solutions to mitigate the multipath fading problem is based on time or frequency diversity both at the transmitter and the receiver. The transmit diversity approach previously proposed by Alamouti [1] seems to be the most realistic scheme which is commonly used in MIMO systems. 
When we are dealing with intercepted signals, the facing problems become more channelling. This scenario is required in various applications such as: Control of civilian authorities over the radio-band frequency, the control of communication quality, warfare, design of universal receiver, etc. For divers reasons, this subject is not well reported in the literature $[2,11]$. In this manuscript, the identification and the separation of OSTBC MIMO transmitted signals, in non data aided context, are addressed.

\section{Mathematical Model \& background}

In this manuscript, the two widely used OSTBC codes are considered:

- Alamouti's code is an OSTBC2 (i.e. $n_{T}=2$ ). Recently, this code has been introduced in many wireless communication standards and systems [16] (WiFi, IEEE $802.11 \mathrm{n}, 4 \mathrm{G}$, etc). Alamouti's code can be implemented using the following coding matrix:

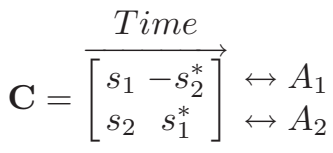

- When $n_{T}=3$, the orthogonal space-time block code is denoted by OSTBC3. Various OSTBC3 can be found in the literature [16]. In our study, one of the most efficient code is considered (i.e. it has the maximum ratio of symbol number $N_{s}=3$ to the transmission period $L=4$ ):

$$
\mathbf{C}=\frac{\text { Time }}{\left[\begin{array}{cccc}
s_{1} & 0 & s_{2} & -s_{3} \\
0 & s_{1} & s_{3}^{*} & s_{2}^{*} \\
-s_{2}^{*} & -s_{3} & s_{1}^{*} & 0
\end{array}\right]} \stackrel{\leftrightarrow A_{1}}{\leftrightarrow A_{2}}
$$

Let us denote by $\mathbf{x}(t)$, a $n_{R} \times 1$ complex vector, the signals received by an antenna array and by $\mathbf{s}=\left(s_{j}(t)\right)$ the signals emitted by $n_{T}$ transmitter antennas. The relationship among the received signals and the emitted signals can be represented by the following equation:

$$
x_{i}(t)=\sum_{j=1}^{n_{T}} h_{i j}(t, \tau) \otimes s_{j}(t)+b_{i}(t)
$$

Here $\otimes$ stands for the convolutive product, $h_{i j}$ denotes the channel impulsive response between the ith emitter and the jth transmitter, and $\mathbf{b}=\left(b_{i}(t)\right)$ represents the noise vector. Hereinafter, many realistic assumptions are made:

- The transmitted signals are mutually independent signals.

- The noises and the signals are independent of each other.

- The noise $\mathbf{b}=\left(b_{i}(t)\right)$ is a zero-mean complex additive white gaussian noise.

- The channel parameters are unknown. However in many wireless applications, the channel can be considered as a quasi-stationary flat fading channel. In this case, one can neglect the Inter-symbol interference (ISI) and simplify the previous model (3) as following [4]:

$$
x_{i}=\sum_{j=1}^{n_{T}} h_{i j} s_{i}+b_{i} \Longrightarrow \mathbf{x}=\mathbf{H} \mathbf{s}+\mathbf{b}
$$


- The receiver number $n_{R}$ can be higher or lower than the emitter number $n_{S}$.

- The Symbol Rate (SR) and Carrier Wave Frequency (CWF) are unknown but they can be estimated $[5,12]$. This subject is beyond the scope of this manuscript, for further details please see $[5,12]$ and their references.

- Using the previous assumption, the received signals can be over-sampled, that means the number of samples per symbol is greater than one. In addition a Carrier Frequency estimation error $\Delta f_{0}$ is introduced in our simulations.

- The emitted and received signals are not synchronize and a random demodulation phase $\phi \in[0,2 \pi]$ is considered.

- An asynchronous reception scheme is assumed. In this case, the sampling process is not necessarily synchronize with the symbol sequence.

\section{Presence detection of OSTBC signals}

The main idea of this manuscript consists in blindly separating OSTBC signals. In order to reach our goal, the OSTBC coding matrices are taken into consideration, this point is addressed in the next section. The latest statement means that a prior information about the transmitted OSTBC signals should be available. The priori information can be considered as a strong assumption. In order to relax that assumption, we briefly describe her our new and simple approach to detect the presence of an OSTBC in the mixed and observed signals.

In order to clarify the main idea, we assume that at most one Alamouticoded signal could be transmitted. The proposed approach can be generalized to deal with similar OSTBC signals. For non-noisy Alamouti-coded signal the eigenvectors of the observed signal covariance matrix $\mathbf{R}_{N \times N}=\mathbf{X X} \mathbf{X}^{\mathbf{H}}=\mathbf{U} \boldsymbol{\Delta} \mathbf{U}^{H}$ are given by the following relationship, see [14]:

$$
\begin{aligned}
& \mathbf{u}_{1}=\mathcal{X}\left(\mathbf{s}_{(\mathbf{1})}, \mathbf{s}_{(\mathbf{2})}\right)=\left(a \mathbf{s}_{(\mathbf{1})}+b e^{-j \theta} \mathbf{s}_{(\mathbf{2})}\right) e^{i \psi_{1}} / \sqrt{\lambda_{1}} \\
& \mathbf{u}_{2}=\mathcal{Y}\left(\mathbf{s}_{(\mathbf{1})}, \mathbf{s}_{(\mathbf{2})}\right)=\left(b \mathbf{s}_{(\mathbf{1})}-a e^{-j \theta} \mathbf{s}_{(\mathbf{2})}\right) e^{i \psi_{2}} / \sqrt{\lambda_{2}}
\end{aligned}
$$

where $\mathbf{s}_{(\mathbf{1})}=\left[s_{1}-s_{2}^{*} \cdots s_{N-1} s_{N}^{*}\right]$ and $\mathbf{s}_{(\mathbf{2})}=\left[s_{2} s_{1}^{*} \cdots s_{N} s_{N-1}^{*}\right]$ stand for the vectors of the transmitted symbols, $a, b, \theta, \psi_{1}$ and $\psi_{2}$ could be any number satisfying the following constraint $a^{2}+b^{2}=1$.

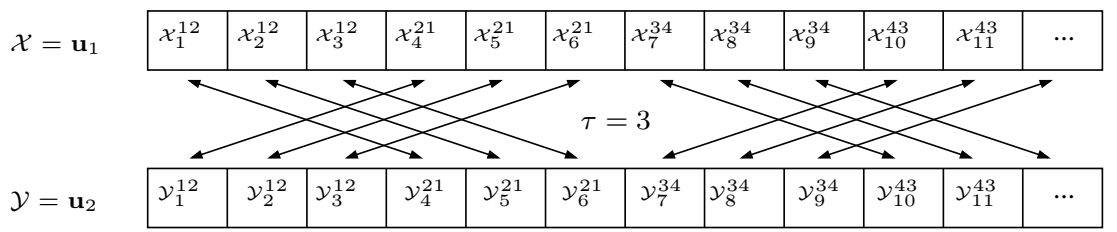

Fig. 1. The cross-correlation of an Alamouti's sequence using 3 samples/symbole, synchronization error $(\tau=3)$. The maximum cross correlation value is only obtained when the two sequence are synchronized. Here, $\mathcal{X}_{k}^{m n}=\mathcal{X}\left(s_{m}, s_{n}\right), \mathcal{X}_{k}^{n m}=\mathcal{X}\left(-s_{n}^{*}, s_{m}^{*}\right)$, $\mathcal{Y}_{k}^{m n}=\mathcal{Y}\left(s_{m}, s_{n}\right), \mathcal{Y}_{k}^{n m}=\mathcal{Y}\left(-s_{n}^{*}, s_{m}^{*}\right)$ and $m<n$.

Fig. 1 shows the main scheme of our approachin which a modified crosscorrelation matrix of the shifted eigenvectors is used:

$$
\Gamma_{\tau}=\frac{1}{N} \sum_{i=0}^{\left[\frac{N}{2 \tau}\right]-1} \sum_{k=0}^{\tau-1} \mathcal{X}_{2 i \tau+k} \mathcal{Y}_{2 i \tau+\tau+k}+\mathcal{Y}_{2 i \tau+k} \mathcal{X}_{2 i \tau+\tau+k}
$$


where $\left[\frac{N}{2 \tau}\right]$ is the integer part of $\frac{N}{2 \tau}$. Using the previous definition, the crosscorrelation is obviously a function of the selected delay between the two vectors, $\tau$ and the synchronization error represents by $i=t_{0} \ldots\left[\frac{N}{2 \tau}\right]-1$, i.e. the number of missed samples. This 3D function is shown in Fig. 2. The maxima obtained in that function stand for the symbol sequence beginnings. In this case, the crosscorrelation function can be used to reduce the synchronization problem as well as to estimate the over-sampling ratio. Using that figure, it can be shown that:

- Two consecutive maximum are separate by 8 samples. One can conclude that the over-sampling ratio is 4 (i.e. 4 samples/symbol).

- The first maximum in Fig. 2 occurs at 5 , that means we missed the beginning of the symbol sequence by 5 samples.

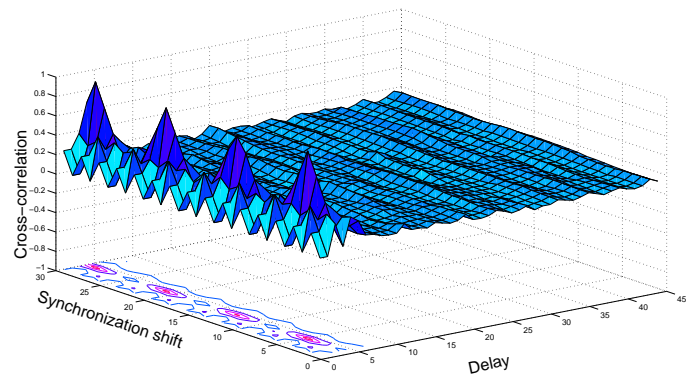

Fig. 2. Eigenvectors cross-correlation modified matrix defined by equation (7).

4 BSS of an underdetermined mixture of OSTBC signals In this section, the separation of underdetermined mixtures of OSTBC signals is addressed. The separation is achieved using the Multi-User Kurtosis (MUK) algorithm and the specific structure of OSTBC coding matrices.

In [8], proposed a BSS algorithm called Multi-User Kurtosis (MUK). MUK achieve the separation by maximizing a contrast function based on the kurtosis. Since the ninetieth of the last century, similar contrast functions have been proposed separately by divers authors [17,3], further details can be founded in [6]. Similar to the previous algorithms, MUK consists of two major steps:

- Orthogonalization and Whiteness.

- Separation achieved by estimating a rotation matrix that maximize the proposed contrast function.

In [8], the orthogonalization process is done using Gram-Schmidt algorithm. By taken into consideration the structure of the OSTBC signals, the orthogonalization procedure can be improved. In our simulations at any iteration, the most modified weight vector is considered and used to establish the weight orthogonal matrix. In the following, the modified MUK algorithm is considered. Fig. 3 shows the effectiveness of the modified version. The comparison shown in the previous figure is obtained by averaging 10000 randomly initialized separations of an Alamouti's coded signals using only one observed signal. As it 


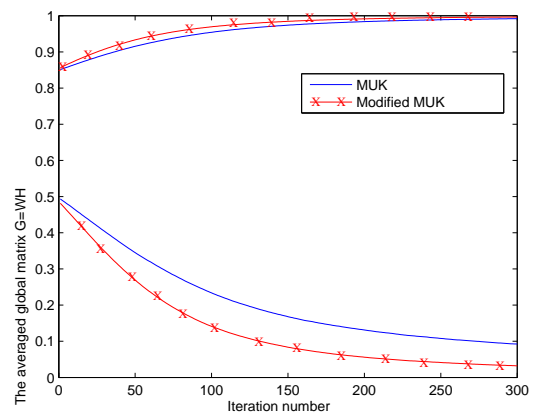

Fig. 3. Comparison between the original and the modified version of MUK.

was mentioned before only two interesting OSTBC signals are studied in this manuscript. The actual study can be straightforward generalized to consider various OSTBC signals. Hereinafter, the transmission channel is assumed to be a Multiple-Input-Single-Output channel (MISO).

\subsection{Extraction of an Alamouti's coded signal}

To transmit an Alamouti's coded signal, two transmission antennas are needed. In the case of noise free channel, the output of the MISO transmission channel is given by the following relationship:

$$
\mathbf{x}=\left[\begin{array}{ll}
h_{1} & h_{2}
\end{array}\right]\left[\begin{array}{ccccccc}
s_{1} & -s_{2}^{*} & s_{3} & -s_{4}^{*} & \ldots & s_{2 N-1} & s_{2 N}^{*} \\
s_{2} & s_{1}^{*} & s_{4} & s_{3}^{*} & \ldots & s_{2 N} & s_{2 N-1}^{*}
\end{array}\right]=\left[\begin{array}{c}
h_{1} s_{1}+h_{2} s_{2} \\
-h_{1} s_{2}^{*}+h_{2} s_{1}^{*} \\
\vdots \\
h_{1} s_{2 N}+h_{2} s_{2 N-1} \\
-h_{1} s_{2 N}^{*}+h_{2} s_{2 N-1}^{*}
\end{array}\right]^{T}
$$

Using the odd and the even components, the observed vector $\mathbf{x}$ can be split into the following two vectors:

$$
\mathbf{x}^{\prime}=\left[\begin{array}{llll}
x_{1} & x_{3} & \ldots & x_{2 N-1} \\
x_{2}^{*} & x_{4}^{*} & \ldots & x_{2 N}^{*}
\end{array}\right]=\left[\begin{array}{cc}
h_{1} & h_{2} \\
h_{2}^{*} & -h_{1}^{*}
\end{array}\right]\left[\begin{array}{cccc}
s_{1} & s_{3} & \ldots & s_{2 N-1} \\
s_{2} & s_{4} & \ldots & s_{2 N}
\end{array}\right]
$$

Without loss of generality, one can assume that the transmitted symbols are Independent and Identically Distributed (iid) [13]. It is obvious that the previous transmission model is similar to an instantaneous complex mixture model.

Many simulations were conducted. Our experimental results shows the effectiveness of the new separation scheme. Fig. 4 shows the separation of a QAM16 transmitted signal using an Alamouti's coding matrix.

\subsection{Extraction of an OSTBC3 signal}

The previous separation scheme can be easily modified to take into consideration another OSTBC coding matrix. In fact, let us consider an OSTBC signal generated using the coding matrix of equation (2). The main key of our separation 

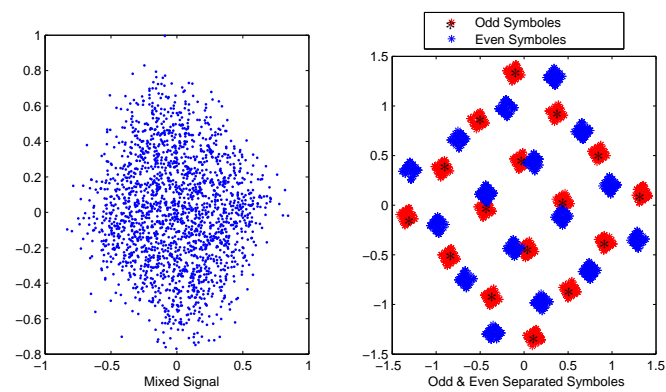

Fig. 4. Extraction of an Alamouti's QAM-16 coded signals using one receiver.

scheme is to find the MISO channel and the Equivalent Virtual Instantaneous mixture (EVI). Using equation (2), one can easily prove that observed signal of a MISO channel should satisfy the following relationship:

$$
\begin{aligned}
& \mathbf{x}=\left[\begin{array}{lll}
h_{1} & h_{2} & h_{3}
\end{array}\right]\left[\begin{array}{ccccccccc}
s_{1} & 0 & s_{2} & -s_{3} & \ldots & s_{3 N-2} & 0 & s_{3 N-1} & -s_{3 N} \\
0 & s_{1}^{*} & s_{3}^{*} & s_{2}^{*} & \ldots & 0 & s_{3 N-2} & s_{3 N}^{*} & s_{3 N-1}^{*} \\
-s_{2}^{*} & -s_{3} & s_{1}^{*} & 0 & \ldots & -s_{3 N-1}^{*} & -s_{3 N} & s_{3 N-2}^{*} & 0
\end{array}\right] \\
& \mathbf{x}^{T}=\left[\begin{array}{c}
x_{1} \\
x_{2} \\
x_{3} \\
x_{4} \\
\vdots \\
x_{4 M-3} \\
x_{4 M-2} \\
x_{4 M-1} \\
x_{4 M}
\end{array}\right]=\left[\begin{array}{c}
h_{1} s_{1}-h_{3} s_{2}^{*} \\
h_{2} s_{1}+h_{3} s_{3} \\
h_{1} s_{2}+h_{2} s_{3}^{*}+h_{3} s_{1}^{*} \\
-h_{1} s_{3}+h_{2} s_{2}^{*} \\
\vdots \\
h_{1} s_{3 N-2}+h_{3} s_{3 N-1} \\
h_{2} s_{3 N-2}+h_{3} s_{3 N} \\
-h_{1} s_{3 N}+h_{2} s_{3 N-1}^{*}
\end{array}\right]
\end{aligned}
$$

In this case, the observed vector should be divided into four sub-vectors using a cyclic selection of its components:

$$
\left[\begin{array}{l}
\mathbf{x}_{(1)} \\
\mathbf{x}_{(2)} \\
\mathbf{x}_{(3)}^{*} \\
\mathbf{x}_{(4)}^{*}
\end{array}\right]=\left[\begin{array}{llll}
x_{1} & x_{5} & \ldots & x_{4 M-3} \\
x_{2} & x_{6} & \ldots & x_{4 M-2} \\
x_{3}^{*} & x_{7}^{*} & \ldots & x_{4 M-1}^{*} \\
x_{4} & x_{8} & \ldots & x_{4 M}
\end{array}\right]=\left[\begin{array}{ccc}
h_{1} & -h_{3} & 0 \\
h_{2} & 0 & -h_{3} \\
h_{3}^{*} & h_{1}^{*} & h_{2}^{*} \\
0 & h_{2} & -h_{1}
\end{array}\right]\left[\begin{array}{cccc}
s_{1} & s_{4} & \ldots & s_{3 N-2} \\
s_{2}^{*} & s_{5}^{*} & \ldots & s_{3 N-1}^{*} \\
s_{3} & s_{6} & \ldots & s_{3 N}
\end{array}\right]=\mathbf{H S}
$$

Using the result of the previous section, one can make the sampling ratio $\rho=1$ sample/symbol. If the transmitted symbol are iid, then the previous model is an EVI model. The separation is done using the modified version of MUK. Fig. 5 shows the separation result of a PSK4 modulated using OSTBC3. In order to conduct that simulation, 2000 symbols, 4 transmission antennas and one receiver are used. We should mention here, that the previous separation scheme can still be applied to extract an OSTBC from a mixed modulated signals when its baud rate is higher or lower than the other ones. The extraction of other signals can 

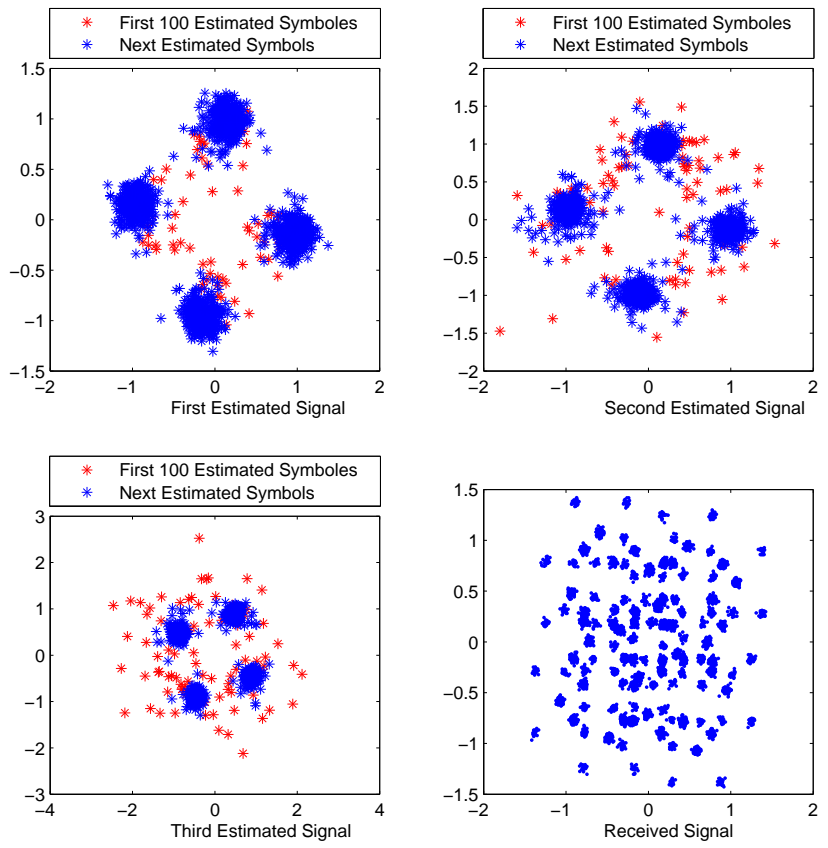

Fig. 5. Extraction of PSK4 symbols transmitted using an OSTBC3

be conducted using a deflation approach. With a high signal to noise ratio, good experimental results are obtained. Due to the lack of space, the details of this part of study is omitted. These points will be the goal of our future studies.

\section{Conclusion}

In this manuscript, the identification, some features extraction and the information retrieval of an OSTBC signals are addressed. In fact, under realistic assumptions, we firstly propose an identification approach based on the crosscorrelation of covariance matrix eigenvalues. In addition, a modified version of Multi-User Kurtosis (MUK) is proposed. Finally, an OSTBC extraction scheme is proposed. The new scheme use the structure of the OSTBC coding matrix and the modified version of MUK.

\section{References}

1. S. Alamouti, "A simple transmit diversity technique for wireless communication," IEEE Journal on Selected Areas in Communications, vol. 16, no. 8, pp. 1451 1458, October 1998.

2. E. E. Azzouz and A. K. Nandi, Automatic modulation recognition of communication signals. Kluwer Academic Publishers, 1996. 
3. N. Delfosse and P. Loubaton, "Adaptive blind separation of independent sources: A deflation approach," Signal Processing, vol. 45, no. 1, pp. 59-83, July 1995.

4. E. G. Larsson and P. Stoica, Space-time block coding for wireless communications. Cambridge: The Press Syndicate of the University of Cambridge, 2003.

5. D. Le Guen and A. Mansour, "Automatic recognition algorithm for digitally modulated signals," in 6th Baiona workshop on signal processing in communications, Baiona, Spain, 25-28 June 2003.

6. A. Mansour, A. Kardec Barros, and N. Ohnishi, "Blind separation of sources: Methods, assumptions and applications." IEICE Transactions on Fundamentals of Electronics, Communications and Computer Sciences, vol. E83-A, no. 8, pp. 1498-1512, August 2000.

7. A. Mansour and M. Kawamoto, "Ica papers classified according to their applications \& performances." IEICE Transactions on Fundamentals of Electronics, Communications and Computer Sciences, vol. E86-A, no. 3, pp. 620-633, March 2003.

8. C. Papadias, "Globally convergent blind source separation based on a multiuser kurtosis maximization criterion," IEEE Trans. on Signal Processing, vol. 48, pp. 3508-3519, December 2000.

9. A. Paulraj, R. Nabar, and D. Gore, Introduction to Space-Time Wireless. New York: Cambridge University Press, 2003.

10. M. Pedzisz and A. Mansour, "Carrier synchronization based on renyi's entropy," in 2004 International Conference on Software, Telecommunications and Computer Networks, Split (Croatia), Dubrovnik (Croatia), Venice (Italy), October 10-13, 2004 2004, pp. 498-501.

11. _ - "Automatic modulation recognition of MPSK signals using constellation rotation and its 4-th order cumulant," Digital Signal Processing, vol. 15, no. 3, pp. 295-304, 2005.

12. —_ "Hos-based multi-component frequency estimation," in 13th European conference on Signal Processing, Theories and Applications (EUSIPCO'2005). Antalya, Turkey: Elsevier, September 2005.

13. J. Proakis, Digital Communications. McGraw-Hill, 1983.

14. M. L. Riediger and P. K.M.Ho, "An eigen-assisted noncoherent receiver for alamouti type space-time modulation," IEEE JOURNAL ON SELECTED AREAS IN COMMUNICATIONS, vol. 33, no. 9, pp. 1811-1820, Sep 2005.

15. V. Tarokh, H. Jafarkhani, and A. R. Calderbank, "Space-time codes high data rate wireless communication: performance crirterion and construction," IEEE Trans. on Information Theory, vol. 44, no. 2, pp. 744-765, March 1998.

16. —_ "Space-time block codes from orthogonal designs," IEEE Trans. on Information Theory, vol. 45, no. 5, pp. 1456-1467, July 1999.

17. E. Weinstein, M. Feder, and A. V. Oppenheim, "Multi-channel signal separation by decorrelation," IEEE Trans. on Speech, and Audio Processing, vol. 1, no. 4, pp. 405-414, October 1993. 